\title{
Práticas alimentares propostas pelo Guia Alimentar para a População Brasileira (2014): cenário após seis anos de seu lançamento
}

\author{
Use Dietary practices proposed by the Dietary Guideline for the Brazilian Population (2014): \\ scenario six years after its launch
}

\author{
Eloysa Lima Silva ${ }^{1} \bullet$, Ingrid Erbert ${ }^{2 *} \odot$ \\ ${ }^{1}$ Graduanda em Nutrição pela Universidade Paulista, São Paulo, São Paulo, Brasil. ${ }^{2}$ Docente na Universidade Paulista, São Paulo, São \\ Paulo, Brasil. Autor para correspondência.E-mail: ierbert@uol.com.br
}

\begin{abstract}
Resumo: Introdução: A sindemia global de excesso de peso demanda políticas púbicas e ações intersetoriais que atuem sobre os determinantes de sociais de saúde. O Guia Alimentar para a População Brasileira (GAPB) publicado em 2014 é instrumento essencial que abrange aspectos culturais, sociais, econômicos e ambientais e sua relação com a alimentação, e apresenta a classificação NOVA dos alimentos. O objetivo do presente estudo foi avaliar o consumo alimentar de indivíduos brasileiros sob a perspectiva das recomendações e classificação NOVA proposta pelo GAPB. Revisão: Realizou-se uma revisão integrativa da literatura, utilizando os descritores 'Guia Alimentar' e os termos 'Food Guide' AND 'Brazil' para busca em inglês. O descritor 'Guia Alimentar' e o filtro 'Brasil' foram utilizados na plataforma SciELO, priorizando pesquisas feitas em território nacional. Na plataforma LILACS, utilizou-se o descritor 'Guia Alimentar' e o filtro compreendendo o período de 2015 a 2020. Na plataforma Cambridge Core utilizaram-se os descritores 'Food Guide' AND 'Brazil' e filtros: tipo de conteúdo, data de publicação e limitação de assunto. Após a adoção dos critérios de elegibilidade, foram selecionados oito estudos. Discussão: Observou-se adequação subótima às práticas alimentares recomendadas pelo GAPB, com o consumo elevado de alimentos ultraprocessados em detrimento aos alimentos in natura. As variáveis classe socioeconômica, renda familiar, demografia, nível de escolaridade, sexo e faixa etária, possuem relação direta com a adoção às práticas alimentares adequadas e saudáveis. O consumo alimentar dos brasileiros está em discordância com as recomendações alimentares propostas pelo GAPB através da classificação NOVA. Considerações finais: O desmonte e o subfinanciamento das políticas públicas de alimentação e nutrição podem dificultar a disseminação das informações do GAPB, reduzindo o potencial das ações de educação alimentar e nutricional especialmente na Atenção Primária à Saúde.
\end{abstract}

Palavras-chave: guia alimentar, Brasil.

\begin{abstract}
Introduction: The global overweight syndemia demands the institution of public policies and intersectoral actions that affects the health determinatives. The Dietary Guideline for the Brazilian Population (DGBP), published at 2014, constitutes an essencial tool and delivers a holistic approach regarding alimentation, dialoguing with cultural, social, economic and environmental facets whilst introducing the NOVA food classification. The objective of this study was to assess the food consumption by Brazilian individuals under the enlightenment of the NOVA food classification in accordance with the DGBP. Review: An integrative review of studies, using the descriptors 'Guia Alimentar' and the terms 'Food Guide’ AND ‘Brazil' for English searches. The descriptor 'Guia Alimentar' and the filter 'Brasil' were used on SciELO database, prioritizing researches done in national territory. On LILACS database, the descriptor 'Guia Alimentar' and the publication interval filter, comprehending a period of five years (2015-2020), were used. On Cambridge Core database the descriptors 'Food Guide’ AND ‘Brazil' and the filters: type of content, publication date and subject limitation were used. After the adoption of the eligibility criteria, eight studies were selected. Discussion: It was observed a suboptimal adequacy of the dietary practices endorsed by DGBP, with the high consumption of ultra-processed aliments to the detriment of the natural ones. The variables socioeconomic class, family income, demographics, scholarship level, gender and age range, have a direct relation with the adoption of healthier and most suitable dietary practices. The food consumption of the Brazilian population is at odds with the dietary recomendations proposed by the DGBP through NOVA food classification. Final considerations: The disassemble and the underfunding of public dietary and nutrition policies may complicate the dissemination of the Guide's messages, diminishing the potential of the Dietary and Nutritional Education actions particularly in the Primary Health Care.
\end{abstract}

Keywords: dietary guideline, Brazil. 


\section{Introdução}

Nas últimas décadas diversos estudos têm consolidado a relação direta entre o consumo de determinados alimentos e a maior ocorrência de doenças crônicas não transmissíveis. A sindemia global de excesso de peso e doenças correlacionadas sinaliza que não é tarefa fácil se alimentar de forma adequada e saudável (Canella et al., 2014).

Torna-se imprescindível a adoção de políticas púbicas e ações intersetoriais que atuem diretamente sobre os determinantes de saúde e por consequência, aqueles que dialogam com a alimentação e nutrição (Brasil, 2014).

Os Guias Alimentares devem facilitar a adoção de escolhas e práticas alimentares mais saudáveis em uma linguagem que seja compreendida por todos, considerando aspectos culturais, sociais, econômicas e ambientais. Constituem-se também como ferramentas educacionais, uma vez que se propõem a apresentar diretrizes oficiais pautadas em ciência, e orientações alimentares capazes de promover saúde e prevenir doenças (Monteiro et al., 2015).

Essas orientações devem ser disseminadas por meio de recomendações de fácil entendimento e compreensão por todas as populações (FAO, 1998).

Devem apontar para um desenvolvimento sustentável e promover políticas e ações voltadas para a Educação Alimentar e Nutricional (EAN), enaltecendo seu potencial emancipatório na adoção de um estilo de vida e de práticas alimentares promotoras de saúde (Monteiro et al., 2015).

A aplicação efetiva dos Guias deve atingir uma abrangência e alcance expandidos dentre a população alvo. Para esse acesso inclusivo, é necessária uma força tarefa de várias estratégias de políticas públicas e programas de diversos setores (públicos e privados), como do Governo Federal, instituições não governamentais, setores privados, da comunidade e da mídia de massa.

É relevante mencionar que a implementação dos Guias Alimentares sempre deve ser seguida, após um determinado período, de algum tipo de avaliação do impacto causado após seu lançamento (FAO, 2007; 2014).

Estudos de avaliação são necessários não somente para verificar mudanças de estilo de vida e de comportamento da população frente às recomendações, mas também como ferramentas de prestação de contas e de elucidação sobre o que efetivamente possui relevância e aplicação, e o que necessita de revisão, bem como fortalecer as evidências de base (Magalhães, 2014).

Na perspectiva brasileira, o primeiro Guia Alimentar para a População Brasileira foi lançado em 2006, destinado à população adulta em geral, trazendo as primeiras diretrizes alimentares oficiais para a população. A criação deste documento objetivava a institucionalização de diretrizes e orientações que pudessem promover a alimentação adequada e saudável proposta pela Política Nacional de Alimentação e Nutrição (PNAN), a Política Nacional de Segurança Alimentar e Nutricional (PNSAN) e a Política Nacional de Promoção da Saúde (PNPS) (Brasil, 2006).

O que se questionou à época era que a lógica de separar os alimentos por grupos alimentares por quantidade de macro/micronutrientes poderia superestimar os nutrientes e alimentos e subestimar e reduzir o nível de processamento industrial ao quais os alimentos são submetidos (Menegassi et al., 2018).

Oliveira et al. (2017) acrescentam que o Guia de 2006 apresentava uma linguagem predominantemente técnica que desestimulava sua leitura pela população em geral.

$\mathrm{O}$ incremento do perfil epidemiológico juntamente à recomendação da OMS resultou no lançamento da segunda edição do Guia Alimentar para a População Brasileira (GAPB), em 2014. O documento possui cinco capítulos e ao final uma sintetização das recomendações, intitulada "Dez Passos Para uma Alimentação Adequada e Saudável”.

Reflexões e opiniões críticas a respeito da versão de 2006 do Guia instigaram pesquisadores como Monteiro et. Al. (2010), que propuseram uma nova classificação dos alimentos -posteriormente denominada classificação NOVA -não mais baseada em grupos alimentares, mas na extensão e propósito de processamento industrial.

Após o aval do Ministério da Saúde (MS) para a inclusão da nova classificação dos alimentos na segunda edição do Guia, a classificação foi aprimorada e passou a conter quatro grupos: alimentos in natura ou minimamente processados, ingredientes, alimentos processados e alimentos ultraprocessados (AUP). O presente estudo teve como objetivo avaliar o consumo alimentar de indivíduos sob a perspectiva das recomendações e da classificação NOVA dos alimentos, em conformidade com o GAPB publicado em 2014. 


\section{Revisão}

Tratou-se de um estudo de revisão integrativa. Portanto, de acordo com o esquema proposto por Mendes et al. (2008), foram selecionados todos os estudos cujos títulos ou resumos avaliassem a adesão das recomendações propostas pelo Guia Alimentar para a População Brasileira.

Para nortear a pesquisa, foi definida a questão: o consumo alimentar dos brasileiros está em consonância com as recomendações do Guia Alimentar para a População Brasileira (Brasil, 2014).

A fim de facilitar a compreensão foi elaborado um fluxograma da seleção amostral dos estudos incluídos no presente estudo.

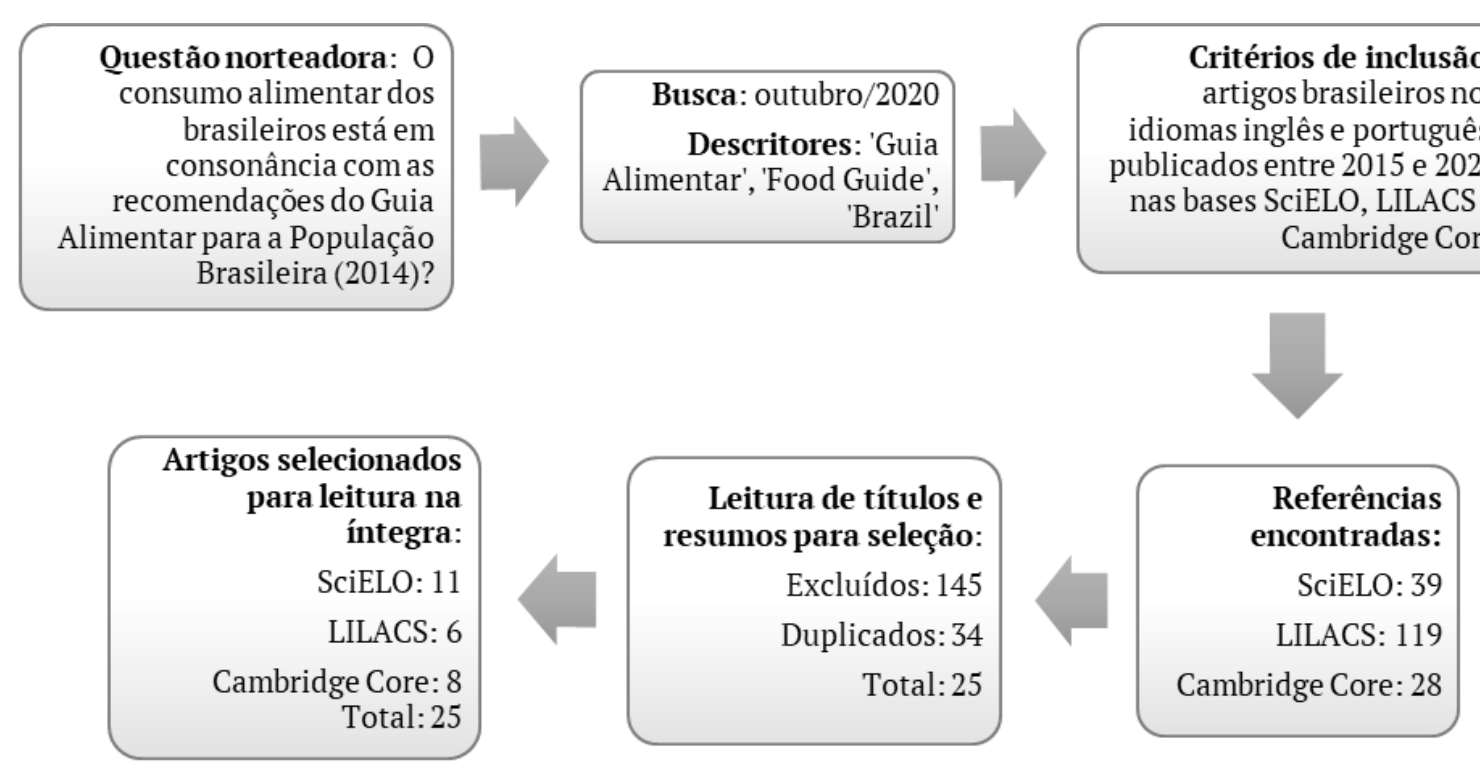

Figura 1. Fluxograma da seleção amostral dos estudos incluídos na revisão integrativa.

\section{Estratégia de busca}

A busca de artigos foi realizada em outubro de 2020. A busca foi feita nas bases de dados Scientific Electronic Library Online (SciELO), LILACS e Cambridge Core, a fim de reconhecer os artigos científicos publicados entre 2015 e 2020, por se tratar de uma avaliação sob a ótica de um material lançado em 2014 (Guia Alimentar para a População Brasileira, $2^{\mathrm{a}}$ edição).

A busca utilizou descritores nos idiomas português e inglês, por meio dos termos 'Guia Alimentar'; e 'Food Guide’ AND ‘Brazil’.

Utilizou-se o descritor ‘Guia Alimentar' e o filtro ‘Brasil' na base de dados SciELO, a fim de obter resultados mais apurados, priorizando as pesquisas feitas em território nacional; além do filtro de data de publicação correspondente ao período entre 2015 e 2020.

Na base de dados LILACS, utilizou-se o descritor 'Guia Alimentar' e o filtro de intervalo de ano de publicação de cinco anos, compreendendo o período de 2015 a 2020.

Na base de dados Cambridge Core utilizaram-se os descritores 'Food Guide' AND 'Brazil', além dos filtros relacionados a: tipo de conteúdo, priorizando os artigos científicos de acesso livre; data de publicação, compreendendo os últimos cinco anos (2015-2020); e limitação de assunto, priorizando o tema nutrição.

Os artigos foram pré-analisados pelos títulos, que deveriam conter referência ao Guia Alimentar e a análise da adesão às suas recomendações, seguido da leitura dos resumos de livre acesso.

\section{Critérios de elegibilidade}

Todo o material obtido pelos sistemas de busca referidos foi submetido aos seguintes critérios de inclusão: serem artigos disponíveis na íntegra (acesso livre), publicados nos periódicos entre 2015 e 2020; propor avaliações de aspectos relacionados às diversas dimensões de alimentação saudável propostas pelo GAPB (comensalidade, consumo de alimentos de acordo com seu grau de processamento, etc); possuir amostra com participantes de nacionalidade brasileira, com idade maior ou igual a 10 anos de idade (correspondendo às faixas etárias de adolescentes e adultos), independentemente do sexo, etnia e nível socioeconômico; e artigos desenvolvidos no Brasil. 
Foram utilizados como critérios de exclusão: teses, trabalhos que não foram publicados no período estipulado e/ou que não estivessem inseridos nas referidas bases de dados, estudos repetidos nas bases, estudos sem acesso livre, além de artigos que não aplicaram algum tipo de avaliação nessa perspectiva, ou seja, que não avaliaram o consumo alimentar ou outras variáveis sob o paradigma adotado no GAPB; e/ou que não foram desenvolvidos no Brasil.

Após a realização da busca segundo as estratégias pré-definidas, foram encontrados 186 artigos, referenciados em três bases de dados: SciELO, LILACS e Cambridge Core, por meio de descritores em inglês e português, publicados entre os anos de 2015 e 2020, sendo encontrados: 39 estudos na base SciELO, 119 artigos na base LILACS e 28 artigos na base Cambridge Core.

Realizou-se leitura crítica e reflexiva dos títulos e resumos encontrados, e deste total encontrado, foram selecionados 25 estudos para leitura na íntegra.

Após leitura sistemática e avaliação da elegibilidade de acordo com critérios de inclusão, exclusão, eliminação dos duplicados e avaliação da qualidade metodológica, estabeleceu-se uma amostra de oito trabalhos.

A Tabela 1 discrimina os artigos quanto: ao autor (es) e ano de publicação do estudo, bem como seu título, tipo de estudo e número amostral, objetivos, principais pontos de interesse (resultados e discussão) e conclusões obtidas.

Tabela 1. Resultados da revisão integrativa sobre a adesão às recomendações do Guia Alimentar para a População Brasileira.

\begin{tabular}{|c|c|c|c|}
\hline Autor(es) /Ano & Título & $\begin{array}{c}\text { Tipo de estudo/ } \\
\text { Amostra (n) } \\
\end{array}$ & Objetivos \\
\hline $\begin{array}{l}\text { Louzada et al. } \\
\qquad(2015)\end{array}$ & $\begin{array}{c}\text { Impacto de } \\
\text { alimentos } \\
\text { ultraprocessados } \\
\text { sobre o teor de } \\
\text { micronutrientes } \\
\text { da dieta no } \\
\text { Brasil. }\end{array}$ & $\begin{array}{c}\text { Transversal, } \\
\text { ( } \mathrm{n}=32.898), \text { critério } \\
\text { de idade } \geqslant 10 \text { anos } \\
\text { de idade. }\end{array}$ & $\begin{array}{l}\text { Avaliar o impacto da } \\
\text { ingestão de AUP sobre } \\
\text { o teor de } \\
\text { micronutrientes na } \\
\text { alimentação da } \\
\text { população brasileira. }\end{array}$ \\
\hline $\begin{array}{l}\text { Araujo et al. } \\
\qquad(2017)\end{array}$ & $\begin{array}{l}\text { Quality of food } \\
\text { choices of } \\
\text { Brazilian } \\
\text { adolescents } \\
\text { according to } \\
\text { individual } \\
\text { earnings. }\end{array}$ & $\begin{array}{c}\text { Transversal, } \\
(\mathrm{n}=3673), \\
\text { adolescentes } 14 \mathrm{e} \\
18 \text { anos. }\end{array}$ & $\begin{array}{c}\text { Avaliar a qualidade das } \\
\text { escolhas alimentares de } \\
\text { adolescentes } \\
\text { brasileiros de acordo } \\
\text { com seus ganhos e } \\
\text { autonomia financeira } \\
\text { individuais. }\end{array}$ \\
\hline
\end{tabular}

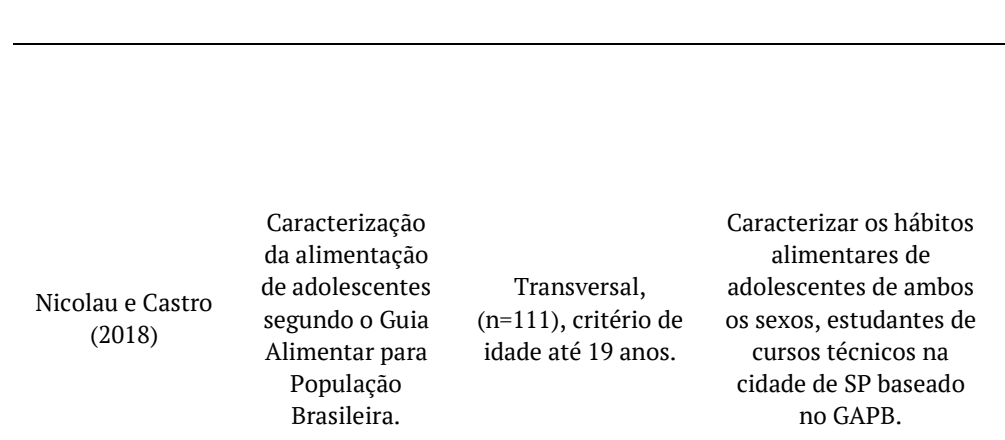

Principais resultados
O consumo calórico médio foi de
1.866 kcal. $69,5 \%$ era composto de
alimentos in natura ou minimamente
processados; 9,0\% de alimentos
processados e $21,5 \%$ de AUP.
Encontrou-se associação negativa
entre consumo de AUP e teor de
micronutrientes para vitaminas B12,
D, E; niacina, piridoxina, cobre,
ferro, fósforo, magnésio, selênio e
zinco. As vitaminas A, C e manganês
não apresentaram associação
significativa entre participação de
AUP e teor do nutriente na dieta.

Conclusões

O consumo de

AUP, quando em
comparação aos in

natura e

minimamente

processados,

impacta

negativamente na

composição de

micronutrientes da

dieta, predispondo

às doenças e

carências

nutricionais.

A renda per capita

A ingestão média de alimentos processados e ultraprocessados aumentaram com o aumento da

renda familiar, enquanto a ingestão

de preparações culinárias diminuiu no mesmo grupo.

O consumo de alimentos in natura,

doces, salgadinhos de pacote e

refrigerantes foi maior no grupo de maior renda familiar.

e renda familiar

foram associados

às escolhas

alimentares. $\mathrm{O}$

consumo de

alimentos

saudáveis e não

saudáveis foi

maior entre $o$

grupo com renda

familiar mais elevada.

$31,5 \%$ dos participantes eram estudantes do curso técnico de Nutrição. 26,1\% relataram omitir o café-da-manhã e 21,6\%, o jantar. Muitos adolescentes não consomem diariamente alimentos in natura ou

minimamente processados. A ingestão diária de frutas é maior do que hortaliças. O consumo diário de AUP foi de 64\%. Menor consumo de arroz e feijão foi associado ao sexo

feminino. O consumo de leite e derivados é significativamente maior no sexo masculino. A participação na compra de alimentos foi associada a menor frequência de consumo diário de refrigerantes.

\begin{tabular}{|c|c|c|c|}
\hline $\begin{array}{l}\text { Alves et al. } \\
\text { (2019) }\end{array}$ & $\begin{array}{c}\text { Avaliação } \\
\text { dietética de } \\
\text { mulheres } \\
\text { Sobreviventes de } \\
\text { Câncer de mama } \\
\text { segundo as } \\
\text { orientações do } \\
\text { Guia Alimentar } \\
\text { para a População }\end{array}$ & $\begin{array}{c}\text { Transversal } \\
(\mathrm{n}=201), \text { sexo } \\
\text { feminino, } \geqslant 18 \\
\text { anos de idade, } \\
\text { diagnosticadas } \\
\text { com câncer de } \\
\text { mama. }\end{array}$ & $\begin{array}{c}\text { Revisar o estado } \\
\text { nutricional de } \\
\text { mulheres pós } \\
\text { tratamento e } \\
\text { diagnóstico de Câncer } \\
\text { de Mama de acordo } \\
\text { com as diretrizes do } \\
\text { GAPB. }\end{array}$ \\
\hline
\end{tabular}
Observou-se no consumo alimentar a predominância de alimentos in natura e minimamente processados. Nenhuma correlação foi observada entre o consumo de alimentos de acordo com seu grau de processamento, e o estado nutricional.

A alimentação não está em consonância com as recomendações do GAPB. 


\begin{tabular}{|c|c|c|c|c|c|}
\hline Autor(es) /Ano & Título & $\begin{array}{c}\text { Tipo de estudo/ } \\
\text { Amostra (n) }\end{array}$ & Objetivos & Principais resultados & Conclusões \\
\hline & & & & & $\begin{array}{c}\text { processados. } \\
\text { Estimou-se que } 1 / 3 \\
\text { da alimentação da } \\
\text { amostra era } \\
\text { composta por } \\
\text { alimentos } \\
\text { processados e } \\
\text { ultraprocessados. }\end{array}$ \\
\hline $\begin{array}{l}\text { Souza et al. } \\
\text { (2019) }\end{array}$ & $\begin{array}{l}\text { Diet Quality } \\
\text { among the } \\
\text { Brazilian } \\
\text { Population and } \\
\text { Associated } \\
\text { Socioeconomic } \\
\text { and } \\
\text { Demographic } \\
\text { Factors: } \\
\text { Analysis from } \\
\text { the National } \\
\text { Dietary } \\
\text { Survey 2008- } \\
2009 .\end{array}$ & $\begin{array}{c}\begin{array}{c}\text { Transversal } \\
(\mathrm{n}=27,760),\end{array} \\
\text { critério de idade } \geqslant \\
18 \text { anos de idade. }\end{array}$ & $\begin{array}{c}\text { Adaptar o HEI-2015 } \\
\text { (Healthy Eating Index) } \\
\text { para a população } \\
\text { brasileira a fim de } \\
\text { avaliar e validar a } \\
\text { confiabilidade do } \\
\text { Îndice de Alimentação } \\
\text { Saudável (IAS) } \\
\text { Adaptado e avaliar a } \\
\text { qualidade da dieta de } \\
\text { adultos brasileiros, } \\
\text { bem como fatores } \\
\text { socioeconômicos e } \\
\text { demográficos } \\
\text { associados à qualidade } \\
\text { da dieta. }\end{array}$ & $\begin{array}{l}\text { O valor de pontuação obtido no IAS } \\
\text { foi de } 45,7 \text { pontos, adequação } \\
\text { subótima às recomendações do } \\
\text { GAPB. } \\
\text { Sexo, idade, nível de escolaridade e } \\
\text { renda per capita foram associados } \\
\text { com qualidade da dieta. } \\
\text { O sexo feminino apresentou } \\
\text { pontuação elevada nas variáveis } \\
\text { relacionadas aos alimentos in natura. } \\
\text { O sexo masculino apresentou } \\
\text { pontuação elevada nas variáveis de } \\
\text { proteínas animais, ácidos graxos, } \\
\text { açúcar de adição e gordura saturada. }\end{array}$ & $\begin{array}{c}\text { O valor de } \\
\text { pontuação obtido } \\
\text { por adultos } \\
\text { brasileiros no IAS } \\
\text { foi de } 45,7 \text { pontos } \\
\text { sugerindo } \\
\text { adequação } \\
\text { subótima às } \\
\text { recomendações do } \\
\text { GAPB. }\end{array}$ \\
\hline $\begin{array}{l}\text { Barbosa et al. } \\
\text { (2020) }\end{array}$ & $\begin{array}{l}\text { Práticas } \\
\text { alimentares de } \\
\text { estudantes } \\
\text { universitários da } \\
\text { área da saúde, } \\
\text { de acordo com } \\
\text { as } \\
\text { recomendações } \\
\text { do Guia } \\
\text { Alimentar para a } \\
\text { População } \\
\text { Brasileira. }\end{array}$ & $\begin{array}{c}\text { Transversal, } \\
(\mathrm{n}=148), \\
\text { estudantes das } \\
\text { áreas de Saúde, } \\
\text { critério de idade } \\
\text { entre } 18 \text { e } 60 \text { anos. }\end{array}$ & $\begin{array}{l}\text { Avaliar as práticas } \\
\text { alimentares de } \\
\text { estudantes } \\
\text { universitários } \\
\text { ingressantes no } 1^{\circ} \\
\text { semestre dos cursos da } \\
\text { área da saúde, de } \\
\text { acordo com as } \\
\text { recomendações do Guia } \\
\text { Alimentar para a } \\
\text { População Brasileira. }\end{array}$ & $\begin{array}{l}\text { As variáveis sociodemográficas (com } \\
\text { exceção do sexo), hábitos de vida e } \\
\text { condições de saúde dos estudantes } \\
\text { não influenciaram nas práticas } \\
\text { alimentares dessa população. } \\
\text { Observou-se predominância de renda } \\
\text { familiar ₹ três salários mínimos, } \\
\text { além de maior inadequação de } \\
\text { práticas alimentares. } \\
\text { Observou-se maior tendência ao } \\
\text { desenvolvimento de práticas } \\
\text { alimentares inadequadas nos } \\
\text { estudantes moradores de república, } \\
\text { em pensão ou com amigos. }\end{array}$ & $\begin{array}{l}\text { As práticas } \\
\text { alimentares dos } \\
\text { universitários } \\
\text { correlacionam-se } \\
\text { positivamente com } \\
\text { a variável “sexo” e } \\
\text { apresentam } \\
\text { elevado percentual } \\
\text { de práticas } \\
\text { alimentares de } \\
\text { risco e } \\
\text { inadequadas. }\end{array}$ \\
\hline $\begin{array}{l}\text { Gabe \& Jaime } \\
\quad(2020)\end{array}$ & $\begin{array}{c}\text { Práticas } \\
\text { alimentares } \\
\text { segundo o Guia } \\
\text { alimentar para a } \\
\text { população } \\
\text { brasileira: } \\
\text { fatores } \\
\text { associados entre } \\
\text { brasileiros } \\
\text { adultos, } 2018 .\end{array}$ & $\begin{array}{c}\text { Transversal, } \\
\text { (n=900), adultos } \\
\text { (18-60 anos de } \\
\text { idade). }\end{array}$ & $\begin{array}{l}\text { Descrever a adequação } \\
\text { de práticas alimentares } \\
\text { às recomendações do } \\
\text { Guia alimentar para a } \\
\text { população brasileira e } \\
\text { analisar fatores } \\
\text { sociodemográficos } \\
\text { associados. }\end{array}$ & $\begin{array}{l}\text { A adequação às recomendações do } \\
\text { Guia esteve diretamente associada } \\
\text { com o aumento da idade e entre } \\
\text { indivíduos das regiões Norte- } \\
\text { Nordeste. } \\
\text { A média de idade dos participantes } \\
\text { foi de 33,5 anos, mulheres e } \\
\text { pertencentes às classes sociais B2 e } \\
\text { C1. Observou-se tendência de } \\
\text { redução da pontuação no sentido das } \\
\text { classes mais altas para as mais } \\
\text { baixas. }\end{array}$ & $\begin{array}{l}\text { A adequação das } \\
\text { práticas } \\
\text { alimentares } \\
\text { baseadas no GAPB } \\
\text { está associada à } \\
\text { idade e região de } \\
\text { moradia. }\end{array}$ \\
\hline $\begin{array}{l}\text { Silva et al. } \\
\text { (2020). }\end{array}$ & $\begin{array}{c}\text { Consumo } \\
\text { alimentar de } \\
\text { gestantes e } \\
\text { fatores } \\
\text { socioeconômico } \\
\text { s associados na } \\
\text { atenção primária } \\
\text { à saúde do } \\
\text { Brasil. }\end{array}$ & $\begin{array}{c}\text { Transversal, } \\
(\mathrm{n}=201) \text { mulheres } \\
\text { grávidas, com } \\
\text { critério de idade } \geqslant \\
19 \text { anos. }\end{array}$ & $\begin{array}{l}\text { Avaliar o consumo } \\
\text { alimentar de gestantes } \\
\text { atendidas na atenção } \\
\text { primária à saúde e } \\
\text { verificar possíveis } \\
\text { associações com } \\
\text { variáveis } \\
\text { socioeconômicas. }\end{array}$ & $\begin{array}{l}\text { A maior parte das gestantes } \\
\text { apresentou idade }<35 \text { anos. } 104 \\
\text { participantes declararam } \\
\text { escolaridade maior do que nove anos, } \\
\text { e a maioria vivia com o parceiro. } \\
154 \text { gestantes possuíam renda } \\
\text { familiar } \leqslant \text { a dois salários mínimos. }\end{array}$ & $\begin{array}{l}\text { A média calórica } \\
\text { de consumo foi } \\
\text { elevada em todos } \\
\text { os grupos } \\
\text { alimentares, com } \\
\text { exceção dos } \\
\text { vegetais. } \\
\text { Gestantes com } \\
\text { menor } \\
\text { escolaridade } \\
\text { consumiram mais } \\
\text { cereais, } \\
\text { tubérculos, raízes } \\
\text { e feijões, porém } \\
\text { demonstraram } \\
\text { menor consumo de } \\
\text { leite e derivados. }\end{array}$ \\
\hline
\end{tabular}

\section{Discussão}

Dentre os estudos apresentados nesta revisão foi observada notável heterogeneidade dos resultados e da natureza das investigações 
A veiculação de estratégias de comunicação de divulgação do Guia é de suma importância e dialoga diretamente com a eficácia do propósito central dos guias. A principal via de divulgação do Guia se dá pelo site do Departamento de Atenção Básica do Ministério da Saúde, que conta com a versão digital do Guia, apesar de versões impressas do Guia terem sido distribuídas em serviços públicos de saúde (Gabe, 2018).

A despeito de pesquisas terem revelado que o acesso à internet no domicílio das populações aumentou, esse fato por si só, já e um fator limitante para a disseminação das recomendações do Guia, uma vez que não garante que as mensagens sejam compreendidas e capazes de sensibilizar os indivíduos acerca da importância da adoção de suas propostas (Oliveira \& Santos, 2020).

Outra possível limitação pode ser a dificuldade por parte de pessoas com dificuldade de leitura e analfabetismo - seja ele funcional ou digital. A Pesquisa Nacional por Amostra de Domicílio Contínua (PNAD Contínua) de 2019 revelou que cerca de 6,8\% de jovens e adultos eram analfabetos (IBGE, 2020a).

Nesse âmbito, diversos materiais complementares ao Guia foram e continuam sendo desenvolvidos, como forma de preencher algumas lacunas decorrentes das diversas limitações que qualquer material educativo pode apresentar. Citam-se como exemplo materiais elaborados pelo Ministério da Saúde, como instrutivos para a realização de ações coletivas em EAN; cadernos de receitas, dentre outros (Gabe, 2018).

Entre 2018 e 2019, outros materiais foram divulgados. Vídeos na plataforma Youtube sobre os capítulos do Guia; um manual prático orientando a implementação do Guia em equipes da Atenção Primária à Saúde (APS); um audiolivro do Guia Alimentar, tornando-o acessível para deficientes visuais; além da elaboração de um Guia em versão de bolso - forma condensada do Guia original - e do teste “Como está a sua alimentação?”, que pretende proporcionar uma autoavaliação dos indivíduos sobre sua alimentação por meio de um questionário qualitativo (Brasil, 2018).

A avaliação de Guias Alimentares tem sido apontada na literatura como uma etapa negligenciada em muitos países, o que pode representar uma fragilidade e dificultar o conhecimento a respeito do real impacto desses instrumentos nas práticas alimentares de populações (FAO, 2014; Keller \& Lang, 2007).

O Guia Alimentar lançado em 2014 já completa seis anos desde o seu lançamento. Torna-se relevante compreender se houve melhora no padrão alimentar das populações na perspectiva do Guia Alimentar. Quantificar essas mudanças pode indicar se/como as informações fornecidas pelo material foram recebidas pelo público-alvo, constatando ou não a eficácia dos objetivos estabelecidos pelo Guia, e a efetividade de suas recomendações.

É consenso que práticas alimentares adequadas e saudáveis, pautadas na preferência do consumo de alimentos in natura e minimamente processados, em locais adequados, preservando a cultura local e a comensalidade, são indispensáveis e pré-determinantes no estado de saúde e qualidade de vida das populações (Louzada et al., 2015).

Dentre os estudos que envolveram apenas adolescentes, foram encontrados resultados que divergem das recomendações nutricionais, como a omissão de refeições, elevado consumo de alimentos ultraprocessados (64\%) e baixo consumo de alimentos in natura e minimamente processados, sendo maior o consumo de frutas do que de vegetais (Nicolau \& Castro, 2018). Em consonância às recomendações do GAPB, o mesmo estudo demonstrou associação direta e positiva entre o consumo regular de arroz e feijão -tradicional composição alimentar brasileira- e a participação em tarefas domésticas relacionadas e o preparo de alimentos.

Em oposição à recomendação desse padrão de alimentação, a mais recente Pesquisa de Orçamentos Familiares (POF) 2017-2018 demonstrou queda na aquisição desses alimentos. Segundo a POF 2017-2018, houve redução de $37 \%$ na aquisição per capita adquirida de arroz, e $52 \%$ de diminuição na compra de feijão, em relação ao período analisado pela POF anterior, entre 2002-2003.

O estudo de Araujo et al. (2017) demonstrou que a autonomia financeira e o aumento dos ganhos individuais dos adolescentes possuia relação direta com o consumo de um marcador de dieta saudável (feijão) e um marcador de dieta não saudável (produtos ultraprocessados e/ou congelados). $\mathrm{O}$ alto consumo de alimentos considerados não saudáveis indica que a autonomia financeira desses adolescentes pode determinar escolhas alimentares menos saudáveis. Ademais, famílias com maior renda demonstraram um consumo três vezes maior desse grupo, quando comparados a famílias de menor poder aquisitivo.

Dados da POF 2017-2018 corroboram com esses achados; a participação no total calórico de alimentos in natura ou minimamente processados e de ingredientes culinários processados diminui com o aumento da renda enquanto os alimentos processados e ultraprocessados aumentam sua participação no total calórico com o aumento da renda (IBGE, 2020b). 
Dentre os estudos que avaliaram somente o sexo feminino (Alves et al., 2019; Silva et al., 2020), destacase o de Silva et al. (2020), que demonstrou correlação entre menores níveis de escolaridade e consumo diário de leite e derivados -considerado marcador de alimentação saudável (Zanchim et al., 2018).

Estudos da Vigilância de Fatores de Risco e Proteção para Doenças Crônicas por Inquérito Telefônico (VIGITEL) 2019 trazem dados semelhantes, demonstrando que o consumo desse tipo de alimento tende a aumentar com a escolaridade. O nível de escolaridade tem sido apontado como variável capaz de interferir na forma como a população escolhe seus alimentos (Moura \& Masquio, 2014).

Apesar de Alves et al. (2019) terem observado que 1/3 da contribuição calórica e de macronutrientes de sua amostra era composta por AUP, ainda não há estudos que estabeleçam limites do consumo de ultraprocessados para a população estudada.

Barbosa et al. (2020) encontraram resultados opostos aos apontados anteriormente. De acordo com sua pesquisa, estudantes universitários do sexo masculino apresentaram maior frequência de práticas alimentares de risco; em contrapartida, as mulheres apresentaram maior frequência de práticas alimentares inadequadas - consideradas neste estudo, como mais prejudiciais.

Gabe e Jaime (2020) correlacionaram maior escore de práticas alimentares adequadas com as variáveis idade, situação socioeconômica e região de moradia. Destacando o fator idade, de acordo com as autoras, a relação direta entre o aumento da idade e a qualidade da alimentação já são bem referenciados na literatura.

Andrade et al. (2016) evidenciaram maiores índices de qualidade da dieta em indivíduos mais velhos, especialmente devido ao aumento do consumo de frutas e diminuição do consumo de gordura saturada e sódio. Com o aumento de idade, a tendência é que as refeições sejam feitas em casa com mais frequência. Além disso, frequentemente podem estar presentes doenças crônicas não transmissíveis que podem motivar melhora no consumo alimentar. Ademais, a transição nutricional também pode explicar os melhores padrões alimentares em indivíduos mais velhos, uma vez que essa transição possui maior impacto nas gerações mais novas (Assumpção et al., 2014).

Louzada et al. (2015) investigaram a qualidade nutricional da alimentação dos brasileiros, avaliando a ingestão diária média per capita dos alimentos, de acordo com a classificação NOVA. Os achados demonstraram que $58,1 \%$ das calorias diárias provinham de alimentos in natura ou minimamente processados, 10,6\% de alimentos processados e 20,4\% de AUP. A participação de alimentos ultraprocessados no consumo alimentar foi associada à densidade energética da dieta e a seu teor de gorduras saturadas, gorduras trans e açúcar livre, e inversamente associada ao teor de fibras, proteínas, vitaminas e minerais, mostrando potencial aumento de risco para obesidade, diabetes, doenças cardiovasculares, doenças relacionadas à deficiência de micronutrientes e alguns tipos de câncer.

Em suma, os estudos aqui apresentados, revelam que a adoção às recomendações e às práticas alimentares preconizadas pelo GAPB ainda se encontram em níveis subótimos; com a substituição dos alimentos in natura/minimamente processados e das refeições caseiras por AUP, bebidas açucaradas e fast-foods com baixa qualidade nutricional.

No Brasil, o GAPB tem um papel estratégico na indução de políticas públicas de alimentação e nutrição e na garantia do direito humano à alimentação e à soberania alimentar. Nesse âmbito, é importante destacar a necessidade de ampla divulgação da classificação NOVA para todo o público ao qual o GAPB se destina, além de ações de ensino e esclarecimento de dúvidas acerca do tema (Menegassi et al., 2018).

Inúmeros são os desafios a serem transpostos nesse campo e vem sendo observado um desmonte das estratégias e políticas públicas de alimentação e nutrição. Dados do Conselho Nacional de Saúde (CNS, 2020) indicam que a aplicação de recursos federais está abaixo da exigência do piso de $15 \%$ previsto na Constituição Federal.

Vasconcelos et al. (2019) supõem que desde 2003, tenha ocorrido um processo de redefinição das estratégias de planejamento, gestão e execução de ações e programas no campo das políticas públicas de alimentação e nutrição, sendo pautados na intersetorialidade e na participação da sociedade civil organizada. Evidências retratam que iniciativas como o Programa Bolsa Família (PBF), trouxeram impacto positivo na redução dos índices de desigualdade de renda e pobreza, diminuição da fome no Brasil, aumento do uso de serviços de saúde por crianças beneficiadas e diminuição da mortalidade infantil pós-neonatal.

A crise econômica global em 2016, que levou ao aumento das taxas de desemprego e intensa recessão, foram agravadas pela crise política vivida no mesmo ano, que culminou na mudança do comando executivo federal, trazendo rupturas institucionais e programáticas e cortes de orçamento que impactaram diretamente 
sob as políticas de alimentação e nutrição, indicando mudanças de paradigma e retrocessos nos princípios e direitos adquiridos (Vasconcelos et al., 2019).

Ademais, a aprovação da Emenda Constitucional 95, que promoveu o congelamento dos pisos de gastos governamentais com saúde e educação por duas décadas, seguramente vem ocasionando o subfinanciamento no repasse de recursos para as políticas e estratégias de alimentação e nutrição (Vasconcelos et al., 2019).

Segundo dados obtidos em Relatórios de Gestão da Coordenação Geral de Alimentação e Nutrição do Departamento de Atenção Básica do Ministério da Saúde (CGAN/DAB/MS) - que tem como missão institucional a coordenação e gestão da PNAN e de suas ações, programas e projetos - os orçamentos e repasses destinados a essas iniciativas sofreram drásticas mudanças.

A organização da atenção nutricional em sistemas de saúde, e em especial, na APS, deve se pautar no reconhecimento do contexto do território no que diz respeito aos seus agravos de saúde relacionados à alimentação e nutrição.

Esse diagnóstico é mais assertivo e efetivo quando instrumentos e sistemas de registro e consolidação de informações são disponibilizados às equipes, de forma que estas possam organizar adequadamente suas ações de promoção, proteção e apoio que contribuam para ampliar as formas de acesso e consumo de uma alimentação mais adequada e saudável. Tais ações, por sua vez, são dependentes de apropriado financiamento e publicação de diretrizes oficiais (Bortolini et al., 2020).

\section{Considerações finais}

Diante dos dados levantados e discutidos no presente estudo, considerou-se que a adoção das recomendações e práticas alimentares sugeridas pelo GAPB ainda não estão em conformidade com o esperado, mesmo após seis anos de seu lançamento. Porém é importante ressaltar que houve avanços importantes em pesquisas, materiais educativos e de apoio à implementação do GAPB na atenção primária, oficinas, dentre outros que contribuem positivamente para a disseminação das recomendações do GAPB.

São necessários mais estudos em diferentes contextos, populações e localidades, sendo sugerida uma pesquisa nacional sobre o tema a partir de uma ferramenta avaliativa adequada ao paradigma do GAPB.

\section{References}

Alves, P. C., Sampaio, H. A. de C., Henriques, E. M. V., Arruda, S. P. M., \& Carioca, A. A. F. 2019. Dietary assessment of women surviving breast cancer according to the Dietary Guidelines for the Brazilian Population. Revista de Nutrição, 32, e180054.

Andrade, S. C., Previdelli, Á. N., Cesar, C. L., Marchioni, D. M., \& Fisberg, R. M. 2016. Trends in diet quality among adolescents, adults and older adults: A population-based study. Preventive medicine reports, 4, 391-396.

Araujo, M., Cunha, D., Bezerra, I., De Castro, M., \& Sichieri, R. 2017. Quality of food choices of Brazilian adolescents according to individual earnings. Public Health Nutrition, 20(17), 3145-3150.

Assumpção, D., Domene, S. M. Á., Fisberg, R. M., \& Barros, M. B. de A. 2014. Qualidade da dieta e fatores associados entre idosos: estudo de base populacional em Campinas, São Paulo, Brasil. Cadernos de Saúde Pública, 30(8), 1680-1694.

Barbosa, B. C. R., Guimarães, N. S., Paula, W. de, \& Meireles, A. L. 2020. Práticas alimentares de estudantes universitários da área da saúde, de acordo com as recomendações do Guia Alimentar para a População Brasileira. Demetra, 15, e45855.

Brasil. Ministério da Saúde. 2018. Secretaria de Atenção Primária à Saúde (SAPS). MS lança conteúdo de divulgação do Guia Alimentar para a População Brasileira. Disponível em: https://aps.saude.gov.br/noticia/5034. Acesso em: 30 mar. 2020.

Brasil. Ministério da Saúde. 2014. Secretaria de Atenção à Saúde. Departamento de Atenção Básica. Guia Alimentar para a População Brasileira (2a ed.). Brasília, DF: Ministério da Saúde.

Brasil. Ministério da Saúde. 2006. Guia alimentar para a população brasileira: promovendo a alimentação saudável. Brasília, DF: Ministério da Saúde.

Bortolini, G. A., de Oliveira, T., da Silva, S. A., Santin, R., de Medeiros, O. L., Spaniol, A. M., Pires, A., Alves, M., \& Faller, L. A. 2020. Ações de alimentação e nutrição na atenção primária à saúde no Brasil. Rev Panam Salud Publica, 44, e39. 
Campos, J. M., Akutsu, R. de C. C. de A., Silva, I. C. R., Oliveira, K. S., \& Monteiro, R. 2020. Gênero, segurança alimentar e nutricional e vulnerabilidade: o Programa das Mulheres Mil em foco. Ciência \& Saúde Coletiva, 25(4), 1529-1538.

Canella, D. S., Levy, R. B., Martins, A. P., Claro, R. M., Moubarac, J. C., Baraldi, L. G., Cannon, G., \& Monteiro, C. A. 2014. Ultra-processed food products and obesity in Brazilian households (2008-2009). PloS one, 9(3), e92752.

Conselho Nacional de Saúde (CNS). Saúde perdeu R\$ 20 bilhões em 2019 por causa da EC 95/2016. 2020. Disponível em: http://www.conselho.saude.gov.br/ultimas-noticias-cns/1044-saude-perdeu-r-20bilhoes-em-2019-por-causa-da-ec-95-2016. Acesso em: 16 nov. 2020.

FAO - Food and Agriculture Organization of the United Nations. 2007. Developing Food-based Dietary Guidelines. A manual from the English- speaking Caribbean.

FAO - Food and Agriculture Organization of the United Nations. 2014. El estado de las guías alimentarias basadas en alimentos en América Latina y el Caribe: 21 ãnos después de la Conferencia Internacional sobre Nutrición.

FAO - Food and Agriculture Organization of the United Nations. 1998. Preparation and Use of Food-Based Dietary Guidelines: report of a joint. FAO/WHO consultation.

Gabe, K.T. 2018. Desenvolvimento e validação de uma escala autoaplicável para avaliação da alimentação segundo as recomendações do Guia Alimentar para a População Brasileira. Tese (Mestrado em Ciências), Faculdade de Saúde Pública da Universidade de São Paulo, São Paulo, 160p. Disponível em: https://www.teses.usp.br/teses/disponiveis/6/6138/tde-08112018-

131030/publico/KamilaTiemannGabeREVISADA_Mtr.pdf. Acesso em: 20 mar. 2020.

Gabe, K. T., \& Jaime, P. C. 2020. Práticas alimentares segundo o Guia alimentar para a população brasileira: fatores associados entre brasileiros adultos, 2018. Epidemiologia e Serviços de Saúde, 29(1), e2019045.

Instituto Brasileiro de Geografia e Estatística [IBGE]. 2020a. Conheça o Brasil. População: Educação. Disponível em: https://educa.ibge.gov.br/jovens/conheca-o-brasil/populacao/18317-educacao.html. Acesso em: 14 out. 2020.

Instituto Brasileiro de Geografia e Estatística [IBGE]. 2020b. Pesquisa de Orçamentos Familiares 2017-2018 $P O F$, Rio de Janeiro, RJ.

Keller, I., \& Lang, T. 2008. Food-based dietary guidelines and implementation: lessons from four countries-Chile, Germany, New Zealand and South Africa. Public health nutrition, 11(8), 867-874.

Louzada, M. L. da C., Martins, A. P. B., Canella, D. S., Baraldi, L. G., Levy, R. B., Claro, R. M., Moubarac, J-C., Cannon, G., \& Monteiro, C. A. 2015. Ultra-processed foods and the nutritional dietary profile in Brazil. Revista de Saúde Pública, 49, 38.

Magalhães, R. 2014. Avaliação de políticas e iniciativas públicas de segurança alimentar e nutricional: dilemas e perspectivas metodológicas. Ciência \& Saúde Coletiva, 19(5), 1339-1346.

Mendes, K. D. S., Silveira, R. C. de C. P., \& Galvão, C. M. 2008. Revisão integrativa: método de pesquisa para a incorporação de evidências na saúde e na enfermagem. Texto \& Contexto - Enfermagem, 17(4), 758-764.

Menegassi, B., Almeida, J. B., Olimpio, M. Y. M., Brunharo, M. S. M., \& Langa, F. R. 2018. A nova classificação de alimentos: teoria, prática e dificuldades. Ciência \& Saúde Coletiva, 23(12), 4165-4176.

Monteiro, C. A., Cannon, G., Moubarac, J. C., Martins, A. P., Martins, C. A., Garzillo, J., Canella, D. S., Baraldi, L. G., Barciotte, M., Louzada, M. L., Levy, R. B., Claro, R. M., \& Jaime, P. C. 2015. Dietary guidelines to nourish humanity and the planet in the twenty-first century. A blueprint from Brazil. Public health nutrition, 18(13), 2311-2322.

Monteiro, C. A., Levy, R. B., Claro, R. M., Castro, I. R. R. de, \& Cannon, G. 2010. A new classification of foods based on the extent and purpose of their processing. Cadernos de Saúde Pública, 26(11), 2039-2049.

Moura, A.F. De. \& Masquio, D.C.L. 2014. A influência da escolaridade na percepção sobre alimentos considerados saudáveis. Rev. Ed. Popular, 13(1), 82-94.

Nicolau, D. A. \& Castro, A. G. P. De. 2018. Characterization of adolescents' diet with the Dietary Guidelines for the Brazilian Population. O Mundo da Saúde, 42(1), 98-122.

Oliveira, M. S. Da S. \& Santos, L. A. Da S. 2020. Guias alimentares para a população brasileira: uma análise a partir das dimensões culturais e sociais da alimentação. Ciência \& Saúde Coletiva, 25(7), 2519-2528.

Oliveira, M.S.S., Amparo-Santos, L., Scagliusi, F.B. 2017. A abordagem da culinária no guia alimentar para a população brasileira. XII Reunión de Antropología del Mercosur. Posadas, Misiones Argentina: RAM. 
Silva, G. S. Da, Carvalho, N. S. De, Abreu, R. B. De, Rocha, B. P., Rodrigues, I. R. Da C., Arruda, S. P. M., Azevedo, D. V. De. 2020. Pregnant women's food consumption and associated socioeconomic factors in Brazil's primary health care. Rev Bras Promoç Saúde, 33, 10587.

Souza, J. de P.M, Lima, M.M de., Horta, P.M. 2019. Diet Quality among the Brazilian Population and Associated Socioeconomic and Demographic Factors: Analysis from the National Dietary Survey 2008-2009. J Acad Nutr Diet., 11, 1866-1874.

Vasconcelos, F. De A. G. De, Machado, M. L., Medeiros, M. A. T. De, Neves, J. A., Recine, E., \& Pasquim, E. M. 2019. Public policies of food and nutrition in Brazil: From Lula to Temer. Revista de Nutrição, 32, e180161.

Zanchim, M. C., Kirsten, V. R., \& Marchi, A. C. B. De. 2018. Marcadores do consumo alimentar de pacientes diabéticos avaliados por meio de um aplicativo móvel. Ciência \& Saúde Coletiva, 23(12), 4199-4208.

\section{Minicurrículo}

Eloysa Lima Silva. Graduanda em Nutrição pela Universidade Paulista, UNIP, SP. Tecnóloga em Gastronomia, formada pelo Centro Universitário SENAC (2016).

Ingrid Erbert. Possui Mestrado em Ensino em Ciências da Saúde pela Universidade Federal de São Paulo (2015), Especialização em Nutrição em Atenção Básica pela Fiocruz (2014), Especialização em Gestão de Sistemas e Serviços de Saúde pela Faculdade de Medicina do ABC (2012), Especialização em Nutrição Clínica pela Universidade Gama Filho (2010), Aprimoramento em Nutrição Hospitalar pelo Centro de Referência de Saúde da Mulher (2009) e graduação em Nutrição pela Universidade Metodista de São Paulo (2008). Atua como docente na Universidade Paulista - UNIP. Possui experiência como docente, preceptora de alunos de especialização e graduação, apoiadora em saúde, nutricionista clínica e NASF.

\footnotetext{
Como citar: Silva, E.L., \& Erbert, I. 2021. Práticas alimentares propostas pelo Guia Alimentar para a População Brasileira (2014): cenário após seis anos de seu lançamento. Pubsaúde, 5, a122. DOI: https://dx.doi.org/10.31533/pubsaude5.a122

Recebido: 31 dez. 2020.

Revisado e aceito: 18 jan. 2021.

Conflito de interesse: os autores declaram, em relação aos produtos e companhias descritos nesse artigo, não ter interesses associativos, comerciais, de propriedade ou financeiros que representem conflito de interesse.

Licenciamento: Este artigo é publicado na modalidade Acesso Aberto sob a licença Creative Commons Atribuição 4.0 (CC-BY 4.0).
} 